\title{
Liver proteomic analysis of postpartum Holstein cows exposed to heat stress or cooling conditions during the dry period
}

\author{
Amy L. Skibiel, ${ }^{*}$ Maya Zachut, $†$ Bruno C. do Amaral, ${ }^{*}$ Yishai Levin, $\ddagger$ and Geoffrey E. Dahl*1 \\ *Department of Animal Sciences, University of Florida, Gainesville 32611 \\ †Department of Ruminant Sciences, Institute of Animal Science, ARO Volcani Center, Rishon Lezion, Israel, 7505101 \\ $\ddagger$ The Nancy and Stephen Grand Israel National Center for Personalized Medicine, Weizmann Institute of Science, Rehovot, Israel, 7610001
}

\section{ABSTRACT}

Heat stress negatively affects cow performance, compromises immune function, and increases susceptibility to metabolic disorders, particularly during the dry period and as cows transition from gestation to lactation. Metabolic adaptations of the liver are critical for successful transition, yet it is unclear how heat stress affects metabolic pathways within the liver at the proteomic level. The objective of this study was to investigate the liver proteome of postpartum cows that were cooled or heat stressed during the dry period to gain insight into how protein expression is altered by prior heat stress and may contribute to performance and disease outcomes. During the dry period, cows were either housed in shaded barns with fans and water soakers [cooled group (CL); $\mathrm{n}=5$ ] or in shaded barns lacking these cooling devices [heat-stressed group (HT); $\mathrm{n}=5]$. Liver biopsies were collected at $2 \mathrm{~d}$ postpartum, and protein content was analyzed by label-free quantitative shotgun proteomics (nanoscale liquid chromatography coupled to tandem mass spectrometry). In the most comprehensive bovine liver proteomics analysis completed to date, we identified 3,270 proteins, 75 of which were differentially expressed between HT and CL cows (fold change \pm 1.2 ). The top pathways differing between HT and CL cows were oxidative phosphorylation, mitochondrial dysfunction, farnesoid $\mathrm{X}$ receptor/ retinoid X receptor (FXR/RXR) activation, and the methylmalonyl pathway. Cooling cows during the dry period likely improves ATP production, reduces oxidative stress, and prevents excessive accumulation of hepatic triglycerides and cholesterol, which may contribute to greater milk yield and lower susceptibility to transition-related diseases.

Key words: oxidative phosphorylation, mitochondria, transition, oxidative stress

Received May 30, 2017.

Accepted September 19, 2017.

${ }^{1}$ Corresponding author: gdahl@ufl.edu

\section{INTRODUCTION}

The transition between late gestation and the onset of lactation is a physiologically challenging process for dairy cows and the predominant time for occurrence of diseases and metabolic disorders (Goff and Horst, 1997; Drackley, 1999). During the periparturient period, increasing energy and nutritional demands for milk production and maintenance needs exceed DMI and a negative energy balance occurs (Bell, 1995; Drackley et al., 2005). To compensate for insufficient nutrient intake, a coordinated suite of physiological adaptations, including enhanced bone resorption, greater intestinal calcium transport, and increased hepatic gluconeogenesis, promotes delivery of substrates to the mammary gland to support milk synthesis (Reynolds et al., 2003; Horst et al., 2005). Furthermore, lower circulating insulin permits fat mobilization from adipose tissue induced by catabolic signaling and spares glucose for milk production (Bell, 1995; Rhoads et al., 2004).

The liver is a central organ coordinating transitionrelated adaptations in lipid, carbohydrate, and protein metabolism. First, circulating nonesterified fatty acids (NEFA) released during fat catabolism from adipose stores are taken up, oxidized, and used to produce ATP in the liver (Reynolds et al., 2003). Additionally, NEFA can be partially oxidized in the liver to produce ketone bodies and used as an alternate energy source in peripheral tissues (Drackley et al., 2005). Excessive NEFA, however, can lead to esterification of triglycerides and storage in the liver as well as greater conversion to ketone bodies, which can result in fatty liver disease and ketosis (Drackley et al., 2005; Schäff et al., 2012). Second, hepatic gluconeogenesis is enhanced, whereby glycerol from fat catabolism, propionate, and amino acids are converted to glucose (Reynolds et al., 2003). Finally, to a lesser extent, amino acids may be converted to pyruvate or tricarboxylic acid cycle intermediates within the liver and used for ATP synthesis (Schäff et al., 2012).

Various management practices, nutrition, and environmental factors, such as heat stress, may impede the 
metabolic shifts necessary for successful transition in otherwise healthy cows (Drackley et al., 2005). Heat stress induces several homeorhetic mechanisms that prioritize thermoregulation over other physiological processes (Baumgard and Rhoads, 2013). As a result, heat-stressed cows have markedly reduced milk production throughout lactation and are more susceptible to metabolic disorders during the transition period (Collier et al., 1982; Kadzere et al., 2002; Bernabucci et al., 2010; Tao and Dahl, 2013). Importantly, adverse effects of heat stress on lactation performance occur not only when cows are exposed to heat stress during lactation, but also when heat stress exposure is confined to the dry period, indicating carry-over effects of environmental stressors in the dry period to the subsequent lactation (Tao and Dahl, 2013). Limited evidence from targeted gene studies indicates that heat stress during the dry period alters mammary gland remodeling and hepatic lipid metabolism (do Amaral et al., 2009, 2011; Tao et al., 2011). For example, mRNA expression of carnitine palmitoyltransferase 1-A $(C P T 1 A)$ and very long chain acyl-CoA dehydrogenase $(A C A D V L)$, which encode proteins involved in fatty acid $\beta$-oxidation, were downregulated in heat-stressed cows at $2 \mathrm{~d}$ postpartum. Nevertheless, relatively little is known about the effect of heat stress during the dry period on other metabolic pathways and hepatic function after calving. The objective of this study was to investigate the liver proteome of postpartum cows that were either cooled or heat stressed during the dry period to gain insight into how molecular pathways are altered by heat stress and may contribute to poor lactation performance and increased incidence of transition-related disorders observed in heat-stressed cows.

\section{MATERIALS AND METHODS}

\section{Experimental Design}

The experiment was conducted at the University of Florida Dairy Unit (Hague, FL) in summer 2008, as described in detail elsewhere (do Amaral et al., 2011). Briefly, cows were dried off $46 \mathrm{~d}$ before expected calving and housed in shaded, sand-bedded, freestall barns. Cows were randomly assigned to 1 of 2 treatments for the duration of the dry period (dry-off to calving); the cooled group ( $\mathbf{C L} ; \mathrm{n}=9$ ) was housed in a barn with fans and soakers, and the heat-stressed group (HT; n $=12$ ) was housed in the same barn but lacked access to these cooling devices. Treatment groups were similar in mature-equivalent milk production in the previous lactation and parity $(1.7 \pm 1.1$ lactations for CL and $1.7 \pm 0.9$ lactations for HT). After calving, all cows were housed in the same shaded, sand-bedded, freestall barn with soakers and fans. Air temperature and humidity in the barns were recorded with Hobo Pro series Temp probes (Onset Computer Corp., Pocasset, MA). The effectiveness of treatments was confirmed; temperature-humidity index between the 2 barns were similar, yet CL cows had lower rectal temperatures and respiration rates throughout the dry period relative to HT cows (do Amaral et al., 2011). Dry cows were fed a TMR once daily at $0900 \mathrm{~h}$ and after calving were fed a TMR for lactating cows twice daily at 0800 and 1200 h. Dry matter intake was recorded daily from dry off to $42 \mathrm{~d}$ postcalving. As reported in do Amaral et al. (2011), DMI of CL cows was higher than that of HT cows around the time of calving but not at any other time during the dry period or after calving and there was a tendency for CL cows to have greater milk yield relative to HT cows.

\section{Liver Biopsy Collection}

Liver tissue was collected at $2 \mathrm{~d}$ postpartum from the right side of the animal through the 10th or 11th intercostal space on an approximate line from the hooks to the elbow. Following sterilization of the area, liver tissue ( 0.5 to $1.5 \mathrm{~g}$ of wet weight) was collected using a stainless steel percutaneous liver biopsy tool (Aries Surgical, Davis, CA). Extracted liver tissue was rinsed with sterile saline, snap frozen in liquid nitrogen, and stored at $-80^{\circ} \mathrm{C}$ until proteomics analysis. Tissue from 10 animals ( 5 per treatment) that were asymptomatic for ketosis, metritis, mastitis, or other health complications was used for proteomics analysis in the current study. Tissue from the remaining $4 \mathrm{CL}$ cows and $7 \mathrm{HT}$ cows were used to assess hepatic mRNA expression in a previous study (do Amaral et al., 2011). Sample size was based on previous studies with similar design in dairy cows (Zachut, 2015; Zachut et al., 2016).

\section{Tissue Preparation for Proteomics Analysis}

Approximately $30 \mathrm{mg}$ of tissue was homogenized in $1 \mathrm{~mL}$ of lysis buffer that comprised $100 \mathrm{~m} M$ Tris- $\mathrm{HCl}$, 4\% SDS, 0.1 $M$ dithiothreitol, $0.2 M$ phenylmethylsulfonyl fluoride, and protease inhibitor cocktail (SigmaAldrich, St. Louis, MO). Tissue was homogenized using 1-mm ceramic beads in a Precellys 24 Bead-Mill Tissue Homogenizer (Bertin Corp., MD) at 5,000 rpm for 15-s intervals, alternating with brief centrifugation $\left(4^{\circ} \mathrm{C}, 10,000 \times g\right)$ until completely homogenized. The homogenate was incubated for $1 \mathrm{~h}$ at $4^{\circ} \mathrm{C}$ followed by centrifugation $\left(4^{\circ} \mathrm{C}, 10 \mathrm{~min}, 13,000 \times g\right)$. The protein phase was transferred to a clean microcentrifuge tube 
and protein concentration was quantified through the Bradford assay (Bradford reagent; Bio-Rad Laboratories, Hercules, CA). The remaining protein was snap frozen in liquid nitrogen and stored at $-80^{\circ} \mathrm{C}$ until proteomics analysis.

Proteomics analysis was conducted at the Weizmann Institute of Science (Rehovot, Israel). Samples were subjected to in-solution tryptic digestion using a modified filter-aided sample preparation protocol (Wiśniewski et al., 2009). Lysates were boiled for $3 \mathrm{~min}$ at $95^{\circ} \mathrm{C}$, and centrifuged for $10 \mathrm{~min}$ at 16,000 $\times \mathrm{g}$. Lysates were mixed with urea buffer ( $8 M$ urea in $0.1 M$ Tris- $\mathrm{HCl})$, and $50 \mu \mathrm{g}$ of protein was loaded onto $30 \mathrm{kDa}$ molecular weight cutoff filters and centrifuged. A second buffer containing $2 M$ urea and $4 \times$ urea buffer was added to the filter unit and centrifuged at $14,000 \times g$ for $40 \mathrm{~min}$. Trypsin $(1 \mu \mathrm{g})$ was added and the samples incubated at $37^{\circ} \mathrm{C}$ overnight. Digested proteins were centrifuged, acidified with trifluoroacetic acid, and stored at $-80^{\circ} \mathrm{C}$ until analysis.

\section{Liquid Chromatography}

Ultra-performance liquid chromatography-MS (UPLC/MS) grade solvents were used for all chromatographic steps. Each sample was loaded using split-less nano-UPLC (10 kpsi nanoAcquity; Waters, Milford, MA). The mobile phases were $(A) \mathrm{H}_{2} \mathrm{O}+0.1 \%$ formic acid, and (B) acetonitrile $+0.1 \%$ formic acid. Desalting of the samples was performed online using a reversedphase C18 trapping column (internal diameter of 180 $\mu \mathrm{m}, 20 \mathrm{~mm}$ long, 5 - $\mu \mathrm{m}$ particle size; Waters). The peptides were then separated using a HSS T3 nano-column (internal diameter of $75 \mu \mathrm{m}, 250 \mathrm{~mm}$ long, 1.8- $\mu \mathrm{m}$ particle size; Waters) at $0.35 \mu \mathrm{L} / \mathrm{min}$. Peptides were eluted from the column into the mass spectrometer using the following gradient: $4 \%$ to $35 \% \mathrm{~B}$ in $150 \mathrm{~min}, 35 \%$ to $90 \%$ B in 5 min, maintained at $90 \%$ for 5 min and then back to initial conditions.

\section{Mass Spectrometry}

The nano-UPLC was coupled online through a nanoESI emitter $(10-\mu \mathrm{m}$ tip; New Objective, Woburn, MA) to a quadrupole orbitrap mass spectrometer $(\mathrm{Q}$ Exactive Plus, Thermo Fisher Scientific, Waltham, MA) using a FlexIon nanospray apparatus (Proxeon, Thermo Fisher Scientific). Data were acquired in datadependent acquisition (DDA) mode, using a Top20 method. The MS1 resolution was set to 70,000 (at 400 $\mathrm{m} / z$ ) and maximum injection time was set to $20 \mathrm{~ms}$. The MS2 resolution was set to 17,500 and maximum injection time was $60 \mathrm{~ms}$.

\section{Data Processing and Analysis}

Raw data were processed as described in Shalit et al. (2015). Briefly, raw data were imported into Expressionist software v10.5 (Genedata, Basel, Switzerland) for retention time alignment and peak detection of precursor peptides. A master peak list was generated from all MS/MS events and sent for database searching using Mascot v2.5.1 (Matrix Science, Mount Prospect, IL). Data were searched against the bovine sequences using UniprotKB (http://www.uniprot.org/) appended with 125 common laboratory contaminant proteins. Fixed modification was set to carbamidomethylation of cysteines, and variable modifications were set to oxidation of methionines and deamidation of $\mathrm{N}$ or Q. Search results were then filtered using the PeptideProphet algorithm (Keller et al., 2002) to achieve maximum false discovery rate of $1 \%$ at the protein level. Peptide identifications were imported back to Expressions to annotate identified peaks. Quantification of proteins from the peptide data was performed using an in-house script (Shalit et al., 2015). Proteins were grouped based on shared peptides. In the results section, we refer to protein groups as "proteins." Data were normalized based on the total ion current. Protein abundance was obtained by summing the 3 most intense, unique peptides per protein, unless the protein was identified with 1 or 2 , in which case only those were used to calculate the protein intensity.

\section{Western Blot Validations}

Protein extracts were diluted 1:1 in complete Laemmli buffer (Bio-Rad) with $5 \% \beta$-mercaptoethanol, and equal protein amounts were loaded into Any $\mathrm{kD}$ TGX precast protein gels (12 well; Bio-Rad) for SDS-PAGE. Following electrophoresis, proteins were transferred to either a polyvinylidene fluoride (PVDF) or nitrocellulose membrane depending on detection method used [e.g., enhanced chemiluminescence (ECL) or infrared fluorescence (IR)]. For ECL, the PVDF membrane was blocked for 40 min at room temperature in $5 \%$ blocking solution (Blotting-Grade Blocker; Bio-Rad) with 0.05\% Tween 20 and subsequently incubated overnight at $4{ }^{\circ} \mathrm{C}$ with rabbit anti-peroxiredoxin 3 (1:1,000, \#Ab73349, Abcam, Cambridge, UK). The secondary antibody (goat anti-rabbit IgG-peroxidase; 1:40,000, \#A0545, Sigma-Aldrich) was applied for $1 \mathrm{~h}$ at room temperature followed by application of DuoLux chemiluminescent/ fluorescent peroxidase substrate (Vector Laboratories, Burlingame, CA). For IR, the nitrocellulose membrane was blocked for $1 \mathrm{~h}$ at room temperature in Starting Block (TBS) Blocking Buffer (Thermo Scientific) 
followed by incubation overnight at $4^{\circ} \mathrm{C}$ with rabbit anti-COXIV (COX4; 1:1,000, \#4850T, Cell Signaling Technology, Danvers, MA). The secondary antibody (IRDYE 680RD goat anti-mouse IgG, \#925-32211, LICOR Biosciences, Lincoln, NE) was applied for $1 \mathrm{~h}$ at room temperature.

Antibodies for ECL were diluted in 5\% blocking solution whereas antibodies for IR were diluted in Starting Block (TBS) Blocking Buffer (Thermo Scientific) with $0.1 \%$ Tween 20. For both Western blots, Ponceau S (Sigma-Aldrich) staining was used to visualize transfer efficiency, and mouse anti- $\beta$-actin (1:32,000, \#A2228, Sigma-Aldrich) was used as a loading control. Secondary antibodies for anti- $\beta$-actin were as follows: for ECL, anti-mouse IgG-alkaline phosphatase (1:60,000, \#A1293, Sigma-Aldrich); for IR, IRDYE 800CW goat anti-rabbit IgG, \#925-32211, LI-COR Biosciences). For ECL, DuoLux chemiluminescent/fluorescent alkaline phosphatase substrate (Vector Laboratories) was used to react with the alkaline phosphatase conjugated secondary antibody. Chemiluminescent signal was detected with a digital imaging system (Syngene G:box, Syngene International Ltd., Cambridge, UK) and band density quantified using GeneTools software (Syngene International Ltd.). Fluorescent signal was detected with a near infrared fluorescent imaging system (LICOR Odyssey CLX; LI-COR Biosciences) and band intensity was quantified using Image Studio software (LI-COR Biosciences). Band intensities were normalized to the $\beta$-actin control.

\section{Statistical and Bioinformatics Analyses}

Proteomics data, after logarithmic transformation, were analyzed by Student's $t$-test (Genedata) to examine the effect of treatment (HT vs. CL). Fold change was calculated as the ratio of arithmetic means of the intensities of HT versus CL samples. Proteins were regarded as differential at $P \leq 0.05$ and fold change (FC) \pm 1.2 . Differentially expressed proteins were analyzed through Ingenuity Pathway Analysis (IPA; Qiagen, Valencia, CA) to determine the most relevant pathways, physiological functions, and networks. Differential protein abundances from Western blots were analyzed with PROC TTEST in SAS v. 9.4 (SAS Institute Inc., Cary, NC).

\section{RESULTS}

A total of 3,270 proteins were identified in the bovine liver samples (Supplemental Table S1; https://doi.org/ 10.3168/jds.2017-13258). Of these, 3,059 proteins were categorized based on cellular location and function us- ing the Qiagen IPA software (Figure 1). The majority of proteins were located in the cytoplasm $(\mathrm{n}=1,870)$ and function as enzymes $(\mathrm{n}=997)$. Based on our cutoff criteria $(P \leq 0.05, \mathrm{FC} \pm 1.2), 75$ proteins were differentially expressed between treatment groups. The most relevant pathways affected by dry period heat stress (Figure 2) were hepatic oxidative phosphorylation and mitochondrial dysfunction, but pathways involved in amino acid metabolism (e.g., methylmalonyl pathway and 2-oxobutanoate degradation), lipid and glucose metabolism [e.g., farnesoid X receptor/retinoid X receptor (FXR/RXR) activation and AMP-activated protein kinase signaling], and oxidative stress (e.g., Nrf2-mediated oxidative stress response) were also included.

Abundances of the 9 differentially expressed proteins in the top 2 most relevant pathways, oxidative phosphorylation and mitochondrial dysfunction, were lower in the postpartum liver of HT cows compared with CL cows (Figure 3): cytochrome c oxidase subunit 4 isoform 1 (COX4I1), NADH dehydrogenase $1 \alpha$ subcomplex subunits 10, 11, and 12 (NDUFA10, NDUFA11, NDUFA12), NADH dehydrogenase $1 \beta$ subcomplex subunit 2 and 11 (NDUFB2, NDUFB11), NADH dehydrogenase iron-sulfur protein 8 (NDUFS8), NADH dehydrogenase flavoprotein 2 (NDUFV2), and peroxiredoxin-3 (PRDX3). With the exception of PRDX3, an antioxidant, these proteins comprise subunits of complexes I and IV of the electron transport chain (ETC) and, as such, are involved in energy production in the mitochondria. Differences in expression of COX4I1 and PRDX3 between HT and CL cows were validated through Western blots; expression of both proteins was significantly lower in HT cows than in CL cows $(P<0.05$, Figure 4$)$. In the NRF2-mediated oxidative stress response pathway, ferritin light chain (FTL) was more abundant in the liver of HT cows (FC $=1.90, P=0.03)$ and NADPH-dependent carbonyl reductase 1 (CBR1) was less abundant in the liver of HT cows $(\mathrm{FC}=0.72, P=0.03)$.

In pathways of amino acid metabolism, abundance of 2 proteins, propionyl-CoA carboxylase $\alpha$ chain $(\mathrm{FC}=$ $0.78, P=0.04)$ and propionyl-CoA carboxylase $\beta$ chain $(\mathrm{FC}=0.75, P=0.02)$ were lower in the liver of HT cows than in CL cows. In the case of glucose and lipid metabolic pathways, some proteins were more highly expressed in the liver of HT cows, whereas others were more highly expressed in the liver of CL cows. Specifically, apolipoprotein A-IV (APOA4, FC $=2.23, P=$ 0.04), phosphatidylcholine translocator $\mathrm{ABCB} 4$ ( $\mathrm{FC}=$ $1.43, P=0.04)$, fetuin-B $(\mathrm{FC}=1.99, P=0.02)$, and ELAV-like protein $(\mathrm{FC}=1.25, P<0.01)$ were more highly expressed in the liver of HT cows. In contrast, 
(a)

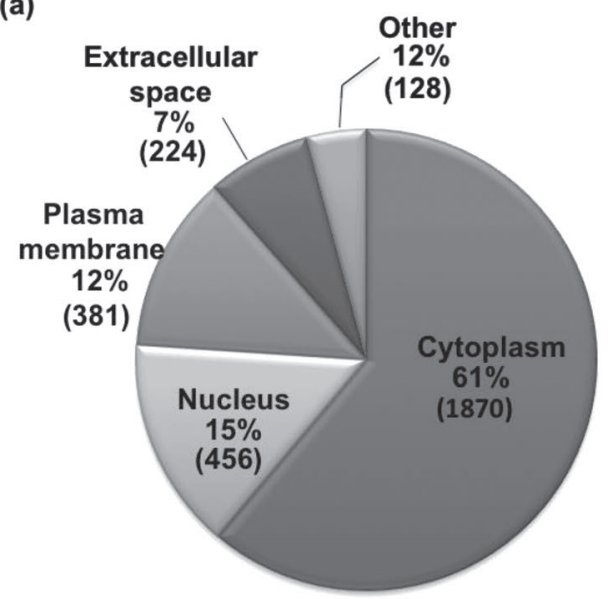

(b)

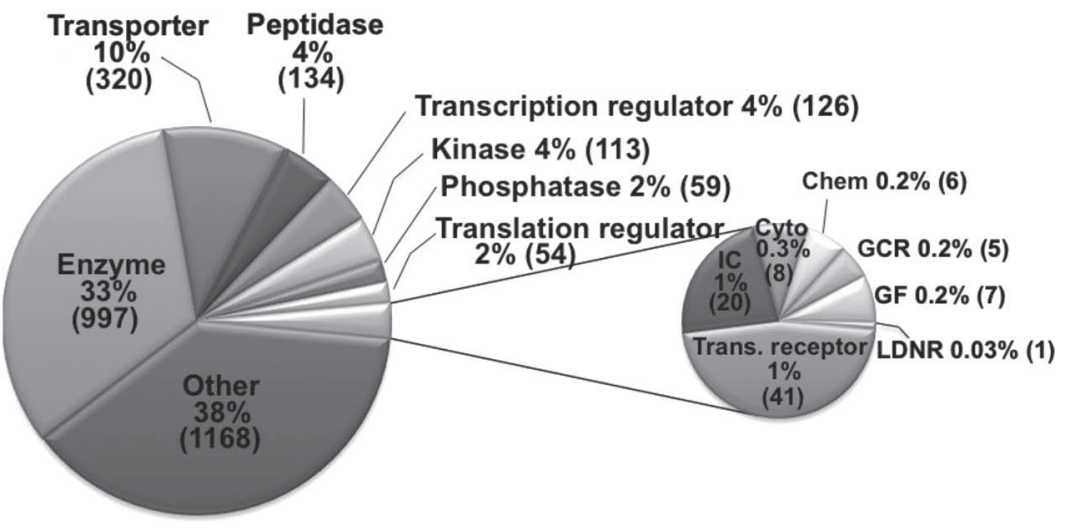

Figure 1. Cellular locations (a) and functions (b) of proteins identified in postpartum bovine liver. Locations and functions of 3,059 proteins were categorized through Ingenuity Pathway Analysis (IPA; Qiagen Inc., Valencia, CA). The number of proteins in each category is provided in parentheses. Trans receptor $=$ transmembrane receptor, $\mathrm{IC}=$ ion channel, Cyto $=$ Cytokine, Chem $=$ chemical-endogenous mammalian, $\mathrm{GCR}$ $=\mathrm{G}$-protein coupled receptor, $\mathrm{GF}=$ growth factor, LDNR = ligand-dependent nuclear receptor.

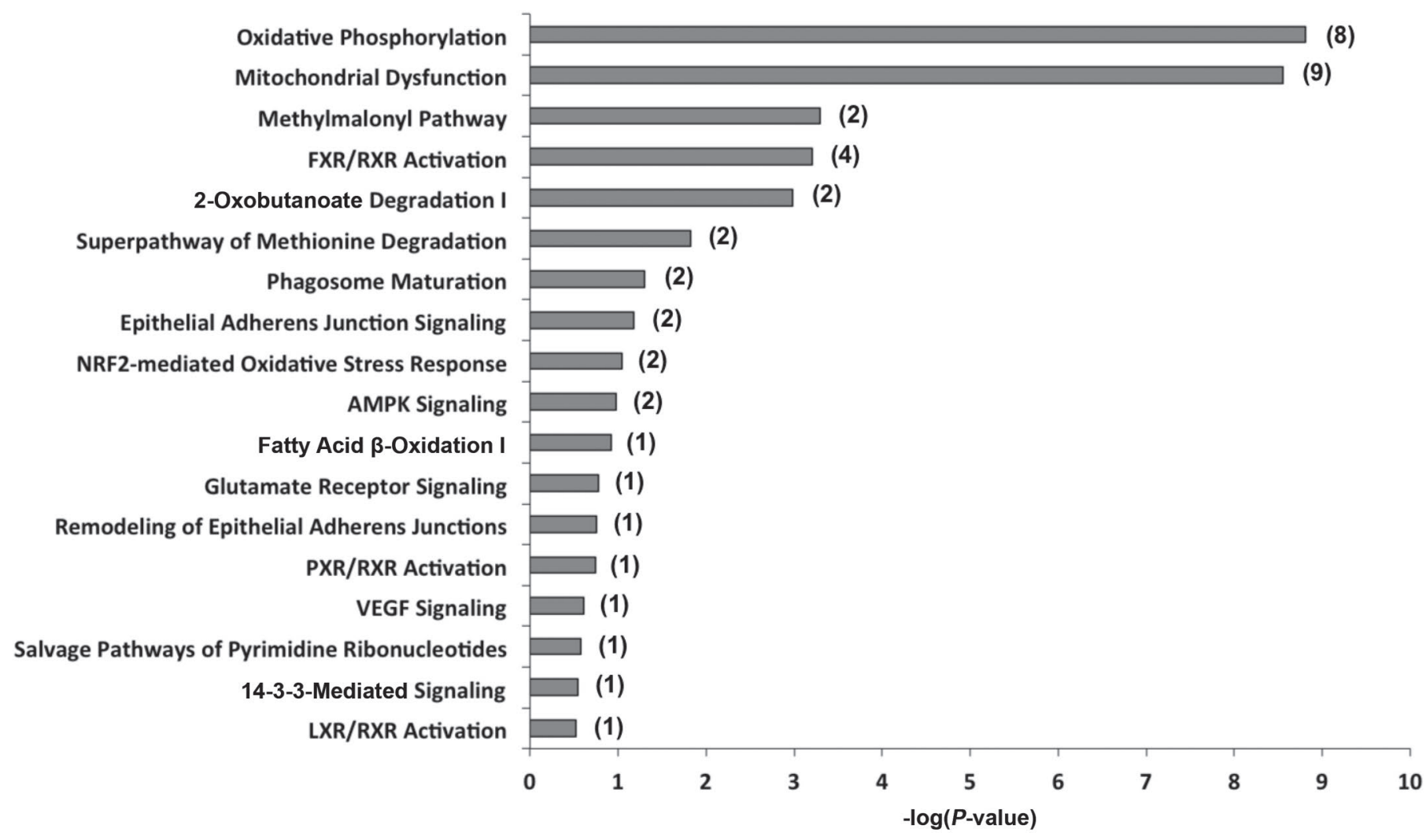

Figure 2. Most relevant molecular and cellular pathways in the postpartum bovine liver affected by heat stress during the dry period. Top affected pathways were identified through Ingenuity Pathway Analysis (IPA; Qiagen Inc., Valencia, CA). Cows were heat stressed (HT) or cooled (CL) during the approximately 46-d dry period, and liver biopsies were collected at $2 \mathrm{~d}$ postpartum. Numbers in parentheses represent the number of proteins in the pathway that are differentially expressed $(P<0.05$ and fold change \pm 1.2$)$ between HT and CL cows. 

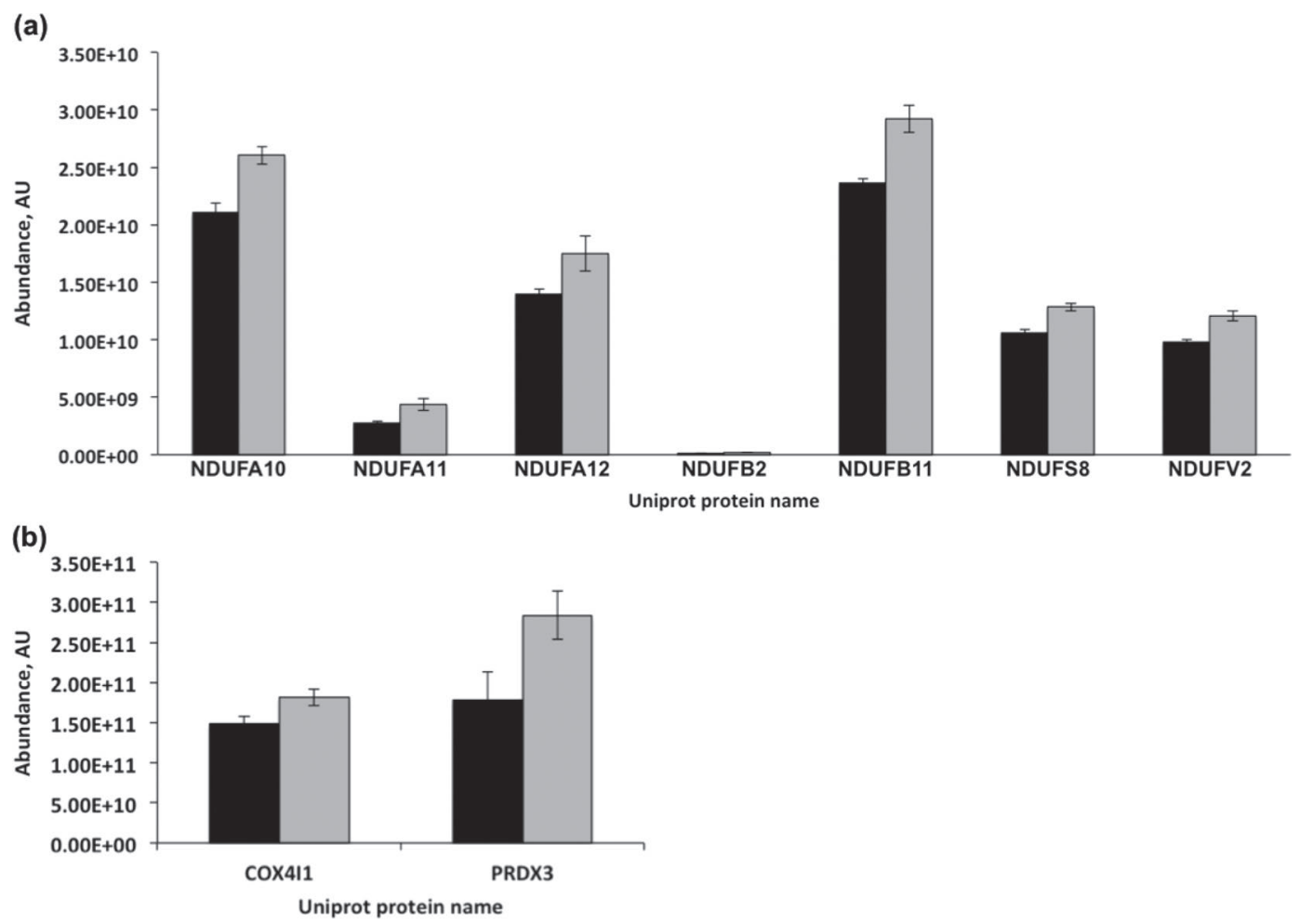

Figure 3. Abundance of proteins in the top 2 most relevant pathways (oxidative phosphorylation and mitochondrial dysfunction) that are differentially expressed between heat-stressed (HT) and cooled cows (CL). (a) Proteins of complex I of the electron transport chain; NDUFS8 and NDUFV2 are core subunits of complex I, and all others are accessory subunits. (b) Subunit of complex IV (COX4I1) of the electron transport chain and an antioxidant (PRDX3). Cows were heat stressed (black bars) or cooled (gray bars) during the approximately 46-d dry period, and liver biopsies were collected at $2 \mathrm{~d}$ postpartum. Data presented as mean abundances. Error bars represent standard errors of the mean. All proteins were lower in abundance in the liver of HT cows than in CL cows $[$ COX4I1, fold change $(\mathrm{FC})=0.82, P<0.04 ;$ NDUFA10, FC $=0.81$, $P<0.01 ;$ NDUFA11, FC $=0.63, P<0.01 ;$ NDUFA12, $\mathrm{FC}=0.80, P<0.04 ;$ NDUFB2, $\mathrm{FC}=0.77, P<0.05 ; \mathrm{NDUFB} 11, \mathrm{FC}=0.81, P<0.01 ;$ NDUFS8, $\mathrm{FC}=0.83, P<0.01 ; \mathrm{NDUFV} 2, \mathrm{FC}=0.81, P<0.01 ; \mathrm{PRDX} 3, \mathrm{FC}=0.63, P<0.04]$.

phosphoenolpyruvate carboxykinase $(\mathrm{FC}=0.74, P<$ $0.01)$ and isovaleryl-CoA dehydrogenase $(\mathrm{FC}=0.83$, $P=0.02)$ were more highly expressed in the liver of CL cows. Consistent with the pathways, biological functions most affected by heat stress involved mitochondrial function, amino acid metabolism, and lipid metabolism (Table 1).

\section{DISCUSSION}

Heat stress during the dry period has many adverse effects on the cow, from suppressing the immune system and reducing feed consumption before calving, to reducing subsequent milk production (Collier et al., 1982; Tao and Dahl, 2013). Successful transition requires the induction of a suite of metabolic adaptations coordinated by the liver that support the energetic demands for copious milk production (Drackley et al., 2005). Although it is well known that heat stress affects these physiological shifts (Rhoads et al., 2009; Wheelock et al., 2010), it is unknown how heat stress affects he- patic metabolism, particularly through the proteome. We identified over 3,000 proteins in the liver of dairy cows at $2 \mathrm{~d}$ postpartum, 75 of which were differentially expressed between cows that were actively cooled or were heat stressed during the dry period. Furthermore, through IPA, we identified the main canonical pathways and biological functions that differed between HT and CL cows.

\section{Oxidative Phosphorylation}

As identified by IPA, top pathways affected by heat stress included oxidative phosphorylation and mitochondrial dysfunction. Oxidative phosphorylation occurs through the ETC, which consists of 5 multimeric protein complexes in the inner mitochondrial membrane that transfer electrons through a series of redox reactions. These reactions result in energy release, which is used to actively transport protons into the intermembrane space of the mitochondria to generate the proton gradient necessary for ATP synthesis by ATP synthase. 

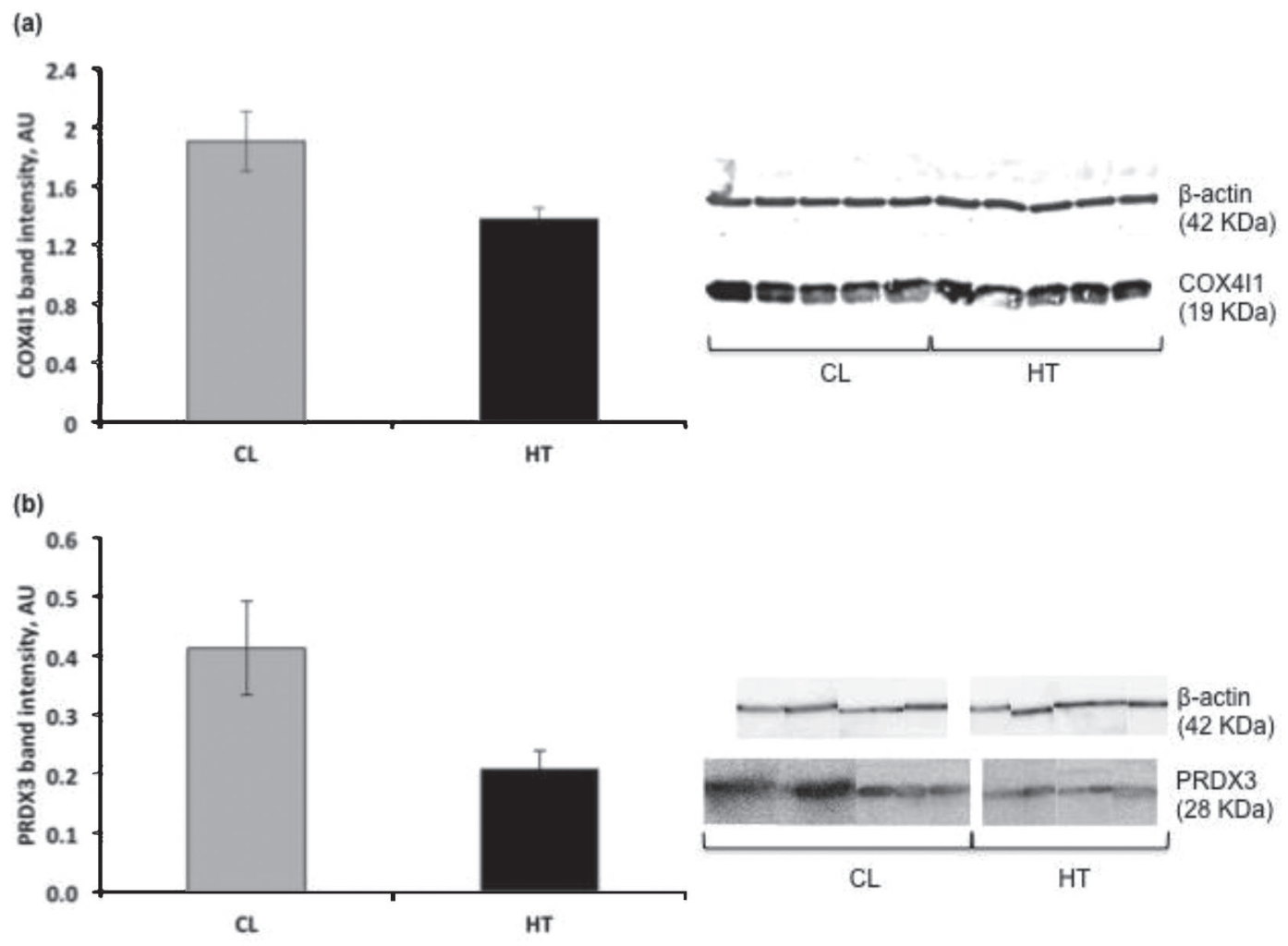

Figure 4. Mean intensity of protein bands validated by Western blot: (a) cytochrome c oxidase subunit 4 isoform 1 (COX4I1), and (b) peroxiredoxin-3 (PRDX3). Band intensity was normalized to $\beta$-actin. Cows were heat stressed (HT; black bars) or cooled (CL; gray bars) during the approximately 46-d dry period, and liver biopsies were collected at $2 \mathrm{~d}$ postpartum. Error bars represent SEM. $P<0.05$ for both proteins.

The catalytic process begins with NADH dehydrogenase (complex I), which oxidizes NADH via flavin mononucleotide and transfers the electrons to ubiquinone through a series of iron-sulfur clusters (Walker, 1992; Lazarou et al., 2009). The final electron transfer occurs via cytochrome c oxidase (complex IV) when electrons are transferred from cytochrome c to oxygen, forming water. Complex I consists of 45 subunits, 14 of which are core subunits obligatory for catalysis and 31 are accessory subunits thought to be involved in assembly, stability, or regulation of complex I or in the defense against oxidative stress (Carroll et al., 2006; Vinothkumar et al., 2014). Complex IV consists of 13 subunits, 3 of which form the catalytic core and 10 that are involved in regulating activity and proton translocation (Kadenbach and Hütteman, 2015).

In the present study, postpartum liver of HT cows had lower expression of NDUFS8 and NDUFV2 proteins, 2 of the 14 core subunits of complex I, but no difference in expression of the core subunits of complex IV. The NDUFV2 protein is involved in the initial oxidation of NADH that begins the process of electron transfer, whereas NDUFS8 is one of the subunits containing an iron-sulfur cluster for downstream electron transfer, ultimately to ubiquinone through the NDUFS7 subunit (Lazarou et al., 2009; Leman et al., 2015). Human patients with mutations affecting core subunits typically have drastically lower ATP production, although the extent of dysfunction appears to be tissue specific. For example, a patient with a mutation affecting NDUFS8 had substantially lower complex I activity in skeletal muscle relative to skin fibroblasts, whereas cardiac muscle lacked activity entirely (Distelmaier et al., 2009).

Besides modifications of the core subunits, complex I and IV activity can also be impaired by defects in accessory subunits, affecting the assembly process or stability of the complex (Lazarou et al., 2009; PagniezMammeri et al., 2012). Human studies of genetic mutations have been instrumental in characterizing the roles of several of the accessory subunits, although the function of most of these subunits is still unclear (Vinothkumar et al., 2014). In contrast to CL cows, HT cows had lower expression of 5 of the 31 complex I accessory subunits, specifically NDUFA10, NDUFA11, NDUFA12, NDUFB2, and NDUFB11, and the COX4 subunit of complex IV. Phosphorylation of the NDUFA10 and NDUFB11 subunits appears to be associated with complex I activity and thus, these subunits are 
Table 1. Most relevant biological functions in the postpartum bovine liver affected by heat stress during the dry period ${ }^{1}$

\begin{tabular}{lcc}
\hline Function annotation & $P$-value & $\begin{array}{c}\text { No. of } \\
\text { molecules }\end{array}$ \\
\hline Hypoplasia & $9.92 \mathrm{E}-03$ & 6 \\
Binding of cells & $4.57 \mathrm{E}-02$ & 5 \\
Anemia & $3.75 \mathrm{E}-02$ & 4 \\
Acidemia & $9.52 \mathrm{E}-06$ & 3 \\
Metabolism of vitamin & $3.54 \mathrm{E}-03$ & 3 \\
Sensitivity of cells & $1.21 \mathrm{E}-02$ & 3 \\
Catabolism of hydrogen peroxide & $2.44 \mathrm{E}-03$ & 2 \\
Mitochondrial function & & \\
Mitochondrial disorder & $4.28 \mathrm{E}-06$ & 6 \\
Mitochondrial complex I deficiency & $3.40 \mathrm{E}-08$ & 5 \\
Protein-related functions & & \\
Organic aciduria & $2.64 \mathrm{E}-07$ & 5 \\
Catabolism of amino acids & $4.86 \mathrm{E}-05$ & 4 \\
Translation of protein & $1.22 \mathrm{E}-02$ & 4 \\
Processing of RNA & $1.98 \mathrm{E}-02$ & 4 \\
Inborn error of amino acid & $5.33 \mathrm{E}-03$ & 3 \\
$\quad$ metabolism & & \\
Homo-oligomerization of protein & $8.33 \mathrm{E}-03$ & 3 \\
Translation of mRNA & $1.97 \mathrm{E}-02$ & 3 \\
Maturation of tRNA & $7.84 \mathrm{E}-06$ & 2 \\
Processing of tRNA & $2.17 \mathrm{E}-04$ & 2 \\
Lipid-related functions & & \\
Catabolism of fatty acid & $2.26 \mathrm{E}-03$ & 2 \\
Absorption of cholesterol & $6.87 \mathrm{E}-03$ & 2 \\
Metabolism of cholesterol & $4.59 \mathrm{E}-02$ & 2 \\
\hline Biogica &
\end{tabular}

${ }^{1}$ Biological functions were identified through Ingenuity Pathway Analysis (Qiagen Inc., Valencia, CA). Cows were heat stressed or cooled during the approximately $46-\mathrm{d}$ dry period. Liver biopsies were collected at $2 \mathrm{~d}$ postpartum.

thought to play a regulatory role in activity or assembly (Papa et al., 2012; Morais et al., 2014). Additionally, NDUFB11 and NDUFA11 are important for stability of other subunits in the complex (Andrews et al., 2013). Knockdown experiments of NDUFA12 in human cell lines resulted in an approximately 50\% decrease in complex I activity, associated with impairment of the later stages of complex I assembly (Rak and Rustin, 2014). Thus, a defect in just a single subunit of complex I can affect assembly, stability, and ultimately, function. Regarding COX4, a series of experiments involving mouse cell lines with reduced expression of COX4 subunit showed substantially reduced levels of complex IV, indicating its function in assembly of the complex $(\mathrm{Li}$ et al., 2006). Furthermore, the experimentally manipulated cell lines had lower complex IV activity and lower ATP production, and predominantly used glycolysis for ATP synthesis compared with a control cell line (Li et al., 2006). Together, these studies suggest that the HT cows in our study likely have lower complex I and IV activity, thereby potentially decreasing ATP production and inducing a switch to glycolysis for the majority of ATP synthesis in the liver. However, further research is necessary to determine the magnitude of heat stress effects on ETC function and energy metabolism in the postpartum cow.

\section{Oxidative Stress}

A secondary consequence of ETC impairment is higher production of reactive oxygen species (ROS). The ETC, particularly complex I, is the major source of ROS in the mitochondria (Distelmaier et al., 2009; Hirst, 2013); ROS are produced when electrons escape the electron transfer process and react with oxygen in the mitochondrial matrix (Kim et al., 2006). When ETC activity is inhibited, more ROS can form (Copeland et al., 2002; Balaban et al., 2005). Imbalances between ROS production and oxidative defense mechanisms can result in oxidative damage to lipids, protein, and DNA, which further reduces ETC activity and ATP production and increases ROS formation (Kim et al., 2006; Distelmaier et al., 2009).

Heat stress affected 2 important defense mechanisms against hepatic oxidative stress in our study. First, expression of PRDX3 was lower in the liver of HT compared with CL cows. Peroxiredoxins are a family of antioxidants that inactivate hydrogen peroxide, one of many ROS, and are typically elevated under conditions of oxidative stress (Immenschuh and Baumgart-Vogt, 2005; Oppermann, 2007). Other peroxiredoxins, such as PRDX1, PRDX2, PRDX4, and PRDX6, were detected in liver samples in our study but expression did not differ between treatment groups, suggesting an overall inability of HT cows to activate the peroxiredoxinmediated defense system. Second, the nuclear factor erythroid 2-related factor 2 (Nrf2)-mediated oxidative stress response was altered in the liver of HT compared with CL cows, as was also found in adipose tissue of cows pregnant in summer versus winter (Zachut et al., 2017). The Nrf2-mediated oxidative stress response is a major pathway for cellular protection against oxidative damage; Nrf2 is a transcription factor regulating the expression of numerous antioxidant proteins and cytoprotective enzymes, such as FTL and NADPH-dependent CBR1 (Nguyen et al., 2009; Wu et al., 2011). In the present study, FTL, a subunit of the iron-storing protein ferritin, was more abundant in the liver of HT cows than CL cows. Ferritin plays a key role in regulating the intracellular concentration of free iron ions (Orino et al., 2001), which is imperative because free iron can react with superoxide anions to form ROS through the Fenton reaction (Linn, 1998). In vitro exposure of HeLa cells to hydrogen peroxide increases ferritin mRNA and protein expression and reduces accumulation of ROS, indicating that oxidative stress induces ferritin synthesis (Orino et al., 2001). In contrast to findings for 
FTL, HT cows exhibited lower expression of CRB1, an enzyme that metabolizes reactive carbonyl compounds formed through ROS peroxidation of lipids or glycoxidation (Ellis, 2007; Oppermann, 2007).

Given that there are many other antioxidants, cytoprotective proteins, and oxidative defense strategies besides peroxiredoxin-mediated and Nrf2-mediated oxidative stress responses, the extent to which ROS accumulation and oxidative stress occurs in the liver of HT cows is unclear. However, other empirical studies involving HT dairy cows lend credence to the assertion that HT causes an imbalance between prooxidation and antioxidation. Bernabucci et al. (2002) found that cows calving in summer relative to spring had higher oxidative stress as indicated by enhanced activity of the antioxidants glutathione peroxidase and superoxide dismutase in erythrocytes. Furthermore, cows calving in summer versus winter had higher plasma concentrations of malondialdehyde (MDA), a marker of oxidative stress (Zachut et al., 2017). It is important to note that even small changes in ROS levels can have catastrophic consequences on cell survival and tissue structure and function (Dröge, 2002; Nicholls, 2004). Additionally, elevated ROS production has been implicated in the pathogenesis of numerous diseases (Madamanchi et al., 2005; Lin and Beal, 2006; Rezaie et al., 2007). Heatstressed cows have a higher incidence of transitionrelated diseases (Bernabucci et al., 2010), which may be at least partially attributed to ROS accumulationoxidative defense imbalances.

\section{Lipid and Glucose Metabolism}

Pathways involved in lipid and glucose metabolism, such as AMPK signaling, FXR/RXR activation, and liver X receptor (LXR)/RXR activation, were altered by heat stress, in congruence with a recent proteomics analysis of adipose tissue in summer gestating cows (Zachut et al., 2017). During the transition period, catabolism of lipids from adipose liberates NEFA, which are important substrates for energy production in the liver (Drackley et al., 2005). However, enhanced NEFA uptake by the liver can increase triglyceride formation, potentially resulting in triglyceride and cholesterol accumulation and increasing the risk of hepatic steatosis (Emery et al., 1992). Cholesterol buildup can cause liver damage and is removed mainly by conversion to bile acids, which are also toxic in excess (Kalaany and Mangelsdorf, 2006; Calkin and Tontonoz, 2012). Thus, a homeorhetic balance between hepatic uptake of NEFA and lipid clearance are vital to meeting the energy demands of the cow while minimizing the probability of tissue toxicity and disease.
Energy-sensing AMPK plays a key role in liver metabolism by regulating transcription factors such as peroxisome proliferator-activated receptor $\alpha$ (PPAR $\alpha)$, sterol regulatory element binding protein 1c (SREBP1c), and carbohydrate responsive element binding protein (ChREBP) that control transcription of several lipogenic and lipolytic genes (Uyeda and Repa, 2006; Li et al., 2011; Martínez et al., 2011). Liver X receptor and FXR are nuclear receptors that form heterodimers with RXR and modulate transcription of genes to regulate lipid and sterol metabolism, often in an opposing fashion (Kalaany and Mangelsdorf, 2006). Whereas cholesterol activation of LXR induces transcription of lipogenic genes and increases cholesterol conversion to bile acids, FXR detects bile acids, regulates their levels via a feedback mechanism, and reduces hepatic triglyceride levels (Kalaany and Mangelsdorf, 2006; Calkin and Tontonoz, 2012). Two proteins in these pathways, apolipoprotein A-IV (APOA4) and phosphatidylcholine translocator (ABCB4) were more abundant in the liver of HT relative to CL cows. The lipid-binding protein APOA4 functions in lipid and cholesterol secretion, particularly through very low density lipoproteins (VLDL) (Karathanasis et al., 1986; VerHague et al., 2013), and ABCB4 is a transporter involved in biliary lipid secretion (Zhang and Edwards, 2008; Calkin and Tontonoz, 2012). Given that VLDL synthesis is extremely low in ruminants and that upregulation of APOA4 is increased in several animal models of steatosis, it is likely that HT cows in our study had a buildup of triglycerides and cholesterol in the liver (Drackley et al., 2005; Hanniman et al., 2006; VerHague et al., 2013). As further support for this hypothesis, summertransitioning cows had a higher liver triglyceride concentration in the early postpartum period relative to those calving in spring (Basiricò et al., 2011).

The cause of lipid accumulation in the liver of HT cows remains to be elucidated. Cows heat stressed during the dry period have lower circulating NEFA in the early postpartum period relative to cows cooled during the dry period (do Amaral et al., 2009; Tao et al., 2012), whereas BHB concentration is similar between HT and CL cows (do Amaral et al., 2011), suggesting that depressed hepatic ketogenesis or excessive lipid mobilization from adipose tissue is not occurring in HT cows. Moreover, in the present study, major proteins involved in triglyceride synthesis, fatty acid $\beta$-oxidation, and hepatic NEFA uptake were similar in abundance between HT and CL cows. Likewise, expression of 2 genescarnitine palmitoyltransferase 1-A (CPT1A) and acylcoenzyme A dehydrogenase $(A C A D V L)$-involved in fatty acid oxidation was similar to or upregulated in the liver of early postpartum CL cows compared with 
HT cows (do Amaral et al., 2011). Thus, lipid accumulation does not appear to be attributed to elevated lipogenesis, fatty acid catabolism, or NEFA uptake in the liver of HT cows. These observations, combined with a reported downregulation of genes involved in VLDL secretion among cows calving in summer versus spring (Shahzad et al., 2015), suggest that enhanced APOA4 protein production in the liver of HT cows represents an unsuccessful attempt to compensate for hepatic lipid buildup. Acute feed deprivation of Holstein cows induced similar hepatic responses, including upregulation of cholesterol transport protein and apolipoprotein, to increase cholesterol and triglyceride secretion (Kuhla et al., 2009). Feed intake was also likely a contributing factor in our study because HT cows had lower feed intake than CL cows around the time of parturition (do Amaral et al., 2011).

In addition to their role in lipid metabolism, FXR and LXR are also important modulators of glucose metabolism. Activation of both receptors inhibits expression of gluconeogenic genes, such as peroxisome proliferator-activated receptor- $\gamma$ coactivator-1 (PGC1) and phosphoenolpyruvate carboxykinase $(P C K)$ (Kalaany and Mangelsdorf, 2006; Zhang and Edwards, 2008; Calkin and Tontonoz, 2012). Phosphoenolpyruvate carboxykinase is a rate-limiting enzyme in gluconeogenesis, catalyzing the conversion of oxaloacetate to phosphoenolpyruvate (Yang et al., 2009). In our study, PCK2 was significantly less abundant in the liver of HT cows compared with CL cows, in contrast to other studies that showed no effect of HT on mRNA expression of the $P C K$ gene in lactating Holstein cows and bull calves (O'Brien et al., 2008; Rhoads et al., 2011). Despite the lower PCK2 abundance in HT cows in our study, plasma glucose concentration was similar between HT and CL cows (do Amaral et al., 2011).

\section{Amino Acid Metabolism}

Heat stress during the dry period was associated with altered amino acid metabolism in early lactation, particularly the methylmalonyl pathway, oxobutanoate degradation, and methionine degradation. Further, $\alpha$ and $\beta$ subunits of the enzyme propionyl-CoA carboxylase (PCCA and PCCB) were less abundant in the liver of HT cows. Propionyl-CoA carboxylase catalyzes the conversion of propionyl CoA to methylmalonyl CoA and, as such, is involved in the use of propionate as a substrate for gluconeogenesis (Flavin and Ochoa, 1957; Tanaka et al., 1975). Thus, our HT cows appeared to shift from propionate to other precursors for glucose synthesis. Similarly, cows calving in summer had differences in hepatic synthesis and degradation of several glucogenic amino acids, such as butanoate, valine, and leucine, compared with cows calving in spring (Shahzad et al., 2015). Decreased use of propionate for glucose production may at least partially reflect lower DMI and reduced availability of rumen propionate (Drackley et al., 2005), which are consistent with previous observations of the effects of HT in dry cows (do Amaral et al., 2011; Tao and Dahl, 2013).

\section{CONCLUSIONS}

This proteomics analysis revealed alterations in protein abundance in the liver of transitioning cows exposed to heat stress or cooling conditions during the dry period. Heat-stressed cattle have impaired mitochondrial function and altered lipid, carbohydrate, and amino acid metabolism in the liver, as indicated by the top canonical pathways and biological functions identified by IPA. Based on differential abundance of proteins in these pathways, it seems probable that heat-stressed cows have reduced ATP synthesis, greater oxidative stress, shifts in precursor supply for gluconeogenesis, and accumulation of hepatic lipids in their liver that may contribute to fatty liver disease. Thus, these changes in liver function may be associated with transition-related diseases and poor lactation performance. Overall, our results indicate that cooling dry cows improves liver function during early lactation, and thus provides greater metabolic support for higher milk yields compared with cows that are heat stressed when dry.

\section{ACKNOWLEDGMENTS}

We thank the staff at the University of Florida Dairy Unit for support during the experimental trial. We appreciate the assistance of Stephanie Wohlgemuth, Tracy Scheffler, and Jimena Laporta (all at the University of Florida) with protein extractions and Western blots. Funding for this project was provided through USDA T-STAR and National Institute of Food and Agriculture-Agriculture and Food Research Initiative (NIFA-AFRI) grants (G. E. Dahl; \#2010-34135-21054 and \#2015-67015-23409) and a Volcani Center Young Scientist Grant (M. Zachut).

\section{REFERENCES}

Andrews, B., J. Carroll, S. Ding, I. M. Fearnley, and J. E. Walker. 2013. Assembly factors for the membrane arm of human complex I. Proc. Natl. Acad. Sci. USA 110:18934-18939.

Balaban, R. S., S. Nemoto, and T. Finkel. 2005. Mitochondria, oxidants, and aging. Cell 120:483-495.

Basiricò, L., P. Morera, N. Lacetera, B. Ronchi, A. Nardone, and U. Bernabucci. 2011. Down-regulation of hepatic ApoB100 expression during hot season in transition dairy cows. Livest. Sci. 137:49-57. 
Baumgard, L. H., and R. P. J. Rhoads. 2013. Effects of heat stress on postabsorptive metabolism and energetics. Annu. Rev. Anim. Biosci. 1:311-337.

Bell, A. W. 1995. Regulation of organic nutrient metabolism during transition from late pregnancy to early lactation. J. Anim. Sci. 73:2804-2819.

Bernabucci, U., N. Lacetera, L. H. Baumgard, R. P. Rhoads, B. Ronchi, and A. Nardone. 2010. Metabolic and hormonal acclimation to heat stress in domesticated ruminants. Animal 4:1167-1183.

Bernabucci, U., B. Ronchi, N. Lacetera, and A. Nardone. 2002. Markers of oxidative status in plasma and erythrocytes of transition dairy cows during hot season. J. Dairy Sci. 85:2173-2179.

Calkin, A. C., and P. Tontonoz. 2012. Transcriptional integration of metabolism by the nuclear sterol-activated receptors LXR and FXR. Nat. Rev. Mol. Cell Biol. 13:213-224.

Carroll, J., I. M. Fearnley, J. M. Skehel, R. J. Shannon, J. Hirst, and J. E. Walker. 2006. Bovine complex I is a complex of 45 different subunits. J. Biol. Chem. 281:32724-32727.

Collier, R. J., S. G. Doelger, H. H. Head, W. W. Thatcher, and C. J. Wilcox. 1982. Effects of heat stress during pregnancy on maternal hormone concentrations, calf birth weight and postpartum milk yield of Holstein cows. J. Anim. Sci. 54:309-319.

Copeland, W. C., J. T. Wachsman, F. M. Johnson, and J. S. Penta. 2002. Mitochondrial DNA alterations in cancer. Cancer Invest. $20: 557-569$.

Distelmaier, F., W. J. H. Koopman, L. P. van den Heuvel, R. J. Rodenburg, E. Mayatepek, P. H. G. M. Willems, and J. A. M. Smeitink. 2009. Mitochondrial complex I deficiency: from organelle dysfunction to clinical disease. Brain 132:833-842.

do Amaral, B. C., E. E. Connor, S. Tao, J. Hayen, J. Bubolz, and G. E. Dahl. 2009. Heat-stress abatement during the dry period: Does cooling improve transition into lactation? J. Dairy Sci. 92:59885999.

do Amaral, B. C., E. E. Connor, S. Tao, J. Hayen, J. W. Bubolz, and G. E. Dahl. 2011. Heat stress abatement during the dry period influences metabolic gene expression and improves immune status in the transition period of dairy cows. J. Dairy Sci. 94:86-96.

Drackley, J. K. 1999. Biology of dairy cows during the transition period: The final frontier? J. Dairy Sci. 82:2259-2273.

Drackley, J. K., H. M. Dann, G. N. Douglas, N. A. Janovick Guretzky, N. B. Litherland, J. P. Underwood, and J. J. Loor. 2005. Physiological and pathological adaptations in dairy cows that may increase susceptibility to periparturient diseases and disorders. Ital. J. Anim. Sci. 4:323-344.

Dröge, W. 2002. Free radicals in the physiological control of cell function. Physiol. Rev. 82:47-95.

Ellis, E. M. 2007. Reactive carbonyls and oxidative stress: Potential for therapeutic intervention. Pharmacol. Ther. 115:13-24.

Emery, R. S., J. S. Liesman, and T. H. Herdt. 1992. Metabolism of long chain fatty acids by ruminant liver. J. Nutr. 122:832-837.

Flavin, M., and S. Ochoa. 1957. Metabolism of propionic acid in animal tissues: I. Enzymatic conversion of propionate to succinate. J. Biol. Chem. 229:965-979.

Goff, J. P., and R. L. Horst. 1997. Physiological changes at parturition and their relationship to metabolic disorders. J. Dairy Sci. 80:1260-1268

Hanniman, E. A., G. Lambert, Y. Inoue, F. J. Gonzalez, and C. J. Sinal. 2006. Apolipoprotein A-IV is regulated by nutritional and metabolic stress: Involvement of glucocorticoids, HNF-4 $\alpha$, and PGC-1 $\alpha$. J. Lipid Res. 47:2503-2514.

Hirst, J. 2013. Mitochondrial complex I. Annu. Rev. Biochem. 82:551575 .

Horst, R. L., J. P. Goff, and T. A. Reinhardt. 2005. Adapting to the transition between gestation and lactation: Differences between rat, human, and dairy cow. J. Mammary Gland Biol. Neoplasia 10:141-156.

Immenschuh, S., and E. Baumgart-Vogt. 2005. Peroxiredoxins, oxidative stress, and cell proliferation. Antioxid. Redox Signal. 7:768 777 .
Kadenbach, B., and M. Hütteman. 2015. The subunit composition and function of mammalian cytochrome $c$ oxidase. Mitochondrion 24:64-76.

Kadzere, C. T., M. R. Murphy, N. Silanikove, and E. Maltz. 2002. Heat stress in lactating dairy cows: A review. Livest. Prod. Sci. 77:59-91.

Kalaany, N. Y., and D. J. Mangelsdorf. 2006. LXRs and FXRs: The yin and yang of cholesterol and fat metabolism. Annu. Rev. Physiol. 68:159-191.

Karathanasis, S. K., P. Oettgen, I. A. Haddad, and S. E. Antonarakis. 1986. Structure, evolution, and polymorphisms of the human apolipoprotein A4 gene (APOA4). Proc. Natl. Acad. Sci. USA 83:8457-8461.

Keller, A., A. I. Nesvizhskii, E. Kolker, and R. Aebersold. 2002. An explanation of the Peptide Prophet algorithm developed. Anal. Chem. 74:5383-5392.

Kim, G. J., K. Chandrasekaran, and W. F. Morgan. 2006. Mitochondrial dysfunction, persistently elevated levels of reactive oxygen species and radiation-induced genomic instability: A review. Mutagenesis 21:361-367.

Kuhla, B., D. Albrecht, S. Kuhla, and C. C. Metges. 2009. Proteome analysis of fatty liver in feed-deprived dairy cows reveals interaction of fuel sensing, calcium, fatty acid, and glycogen metabolism. Physiol. Genomics. 37:88-98.

Lazarou, M., D. R. Thorburn, M. T. Ryan, and M. McKenzie. 2009. Assembly of mitochondrial complex I and defects in disease. Biochim. Biophys. Acta 1793:78-88.

Leman, G., N. Gueguen, V. Desquiret-Dumas, M. Selma Kane, C. Wettervald, S. Chupin, A. Chevrollier, A.-S. Lebre, J.-P. Bonnefont, M. Barth, P. Amati-Bonneau, C. Verny, D. Henrion, D. Bonneau, P. Reynier, and V. Procaccio. 2015. Assembly defects induce oxidative stress in inherited mitochondrial complex I deficiency. Int. J. Biochem. Cell Biol. 65:91-103.

Li, Y., J.-S. Park, J.-H. Deng, and Y. Bai. 2006. Cytochrome c oxidase subunit IV is essential for assembly and respiratory function of the enzyme complex. J. Bioenerg. Biomembr. 38:283-291.

Li, Y., S. Xu, M. M. Mihaylova, B. Zheng, X. Hou, B. Jiang, O. Park, Z. Luo, E. Lefai, Y. J. Shyy, B. Gao, M. Wierzbicki, T. J. Verbeuren, R. J. Shaw, R. A. Cohen, and M. Zang. 2011. AMPK phosphorylates and inhibits SREBP activity to attenuate hepatic steatosis and atherosclerosis in diet-induced insulin-resistant mice. Cell Metab. 13:376-388

Lin, M. T., and M. F. Beal. 2006. Mitochondrial dysfunction and oxidative stress in neurodegenerative diseases. Nature 443:787-795.

Linn, S. 1998. DNA damage by iron and hydrogen peroxide in vitro and in vivo. Drug Metab. Rev. 30:313-326.

Madamanchi, N. R., A. Vendrov, and M. S. Runge. 2005. Oxidative stress and vascular disease. Arterioscler. Thromb. Vasc. Biol. $25: 29-38$.

Martínez, N., V. White, M. Kurtz, R. Higa, E. Capobainco, and A. Jawerbaum. 2011. Activation of hte nuclear receptor PPAR $\alpha$ regulates lipid metabolism in foetal liver from diabetic rats: Implications in diabetes-induced foetal overgrowth. Diabetes Metab. Res. Rev. 27:35-46.

Morais, V. A., A. Grünewald, and B. De Strooper. 2014. Mitochondrial complex I activity via NDUFA10 ubiquinone uncoupling. Science 344:203-207.

Nguyen, T., P. Nioli, and C. B. Pickett. 2009. The Nrf2-antioxidant response element signaling pathway and its activation by oxidative stress. J. Biol. Chem. 284:13291-13295.

Nicholls, D. G. 2004. Mitochondrial membrane potential and aging. Aging Cell 3:35-40.

O'Brien, M. D., L. C. Cole, J. B. Wheelock, S. R. Sanders, G. C. Duff, L. H. Baumgard, and R. P. Rhoads. 2008. Thermal and nutritional regulation of hepatic gluconeogenic genes in growing beef cattle. J. Dairy Sci. 92(E-Suppl. 1):455. (Abstr.)

Oppermann, U. 2007. Carbonyl reductases: The complex relationships of mammalian carbonyl- and quinone-reducing enzymes and their role in physiology. Annu. Rev. Pharmacol. Toxicol. 47:293-322. 
Orino, K., L. Lehman, Y. Tsuji, H. Ayaki, S. V. Torti, and F. M. Torti. 2001. Ferritin and the response to oxidative stress. Biochem. J. 357:241-247.

Pagniez-Mammeri, H., M. Rak, A. Legrand, P. Bénit, P. Rustin, and A. Slama. 2012. Mitochondrial complex I deficiency of nuclear origin II. Non-structural genes. Mol. Genet. Metab. 105:173-179.

Papa, S., D. De Rasmo, Z. Technikova-Dobrova, D. Panelli, A. Signorile, S. Scacco, V. Petruzzella, F. Papa, G. Palmisano, A. Gnoni, L. Micelli, and A. M. Sardanelli. 2012. Respiratory chain complex I, a main regulatory target of the $\mathrm{cAMP} / \mathrm{PKA}$ pathway is defective in different human diseases. FEBS Lett. 586:568-577.

Rak, M., and P. Rustin. 2014. Supernumerary subunits NDUFA3, NDUFA5, and NDUFA12 are required for the formation of the extramembrane arm of human mitochondrial complex I. FEBS Lett. 588:1832-1838.

Reynolds, C. K., P. C. Aikman, B. Lupoli, D. J. Humphries, and D. E. Beever. 2003. Splanchnic metabolism of dairy cows during the transition from late gestation through early lactation. J. Dairy Sci. 86:1201-1217.

Rezaie, A., R. D. Parker, and M. Abdollahi. 2007. Oxidative stress and pathogenesis of inflammatory bowel disease: An epiphenomenon or the cause? Dig. Dis. Sci. 52:2015-2021.

Rhoads, M. L., R. P. Rhoads, M. J. VanBaale, R. J. Collier, S. R. Sanders, W. J. Weber, B. A. Crooker, and L. H. Baumgard. 2009. Effects of heat stress and plane of nutrition on lactating Holstein cows: Production, metabolism, and aspects of circulating somatotropin. J. Dairy Sci. 92:1986-1997.

Rhoads, R. P., J. W. Kim, B. J. Leury, L. H. Baumgard, N. Segoale, S. J. Frank, D. E. Bauman, and Y. R. Boisclair. 2004. Insulin increases the abundance of the growth hormone receptor in liver and adipose tissue of periparturient dairy cows. J. Nutr. 134:1020-1027.

Rhoads, R. P., A. J. La Noce, J. B. Wheelock, and L. H. Baumgard 2011. Alterations in expression of gluconeogenic genes during heat stress and exogenous bovine somatotropin administration. J. Dairy Sci. 94:1917-1921.

Schäff, C., S. Börner, S. Hacke, U. Kautzsch, D. Albrecht, H. M. Hammon, M. Röntgen, and B. Kuhla. 2012. Increased anaplerosis, TCA cycling, and oxidative phosphorylation in the liver of dairy cows with intensive body fat mobilization during early lactation. J. Proteome Res. 11:5503-5514.

Shahzad, K., H. Akbar, M. Vailati-Riboni, L. Basiricò, P. Morera, S. L. Rodriguez-Zas, A. Nardone, U. Bernabucci, and J. J. Loor. 2015. The effect of calving in the summer on the hepatic transcriptome of Holstein cows during the peripartal period. J. Dairy Sci. 98:5401-5413.

Shalit, T., D. Elinger, A. Gabashvili, A. Savidor, and A. Levin. 2015. MS1-based label-free proteomics using a quadrupole orbitrap mass spectrometer. J. Proteome Res. 14:1979-1986.

Tanaka, K., I. M. Armitage, H. S. Ramsdell, Y. E. Hsia, S. R. Lipsky, and L. E. Rosenberg. 1975. [13C] Valine metabolism in methylma- lonicacidemia using nuclear magnetic resonance: Propinonate as an obligate intermediate. Proc. Natl. Acad. Sci. USA 72:3692-3696.

Tao, S., and G. E. Dahl. 2013. Heat stress effects during late gestation on dry cows and their calves. J. Dairy Sci. 96:4079-4093.

Tao, S., J. W. Bubolz, B. C. do Amaral, I. M. Thompson, M. J. Hayen, S. E. Johnson, and G. E. Dahl. 2011. Effect of heat stress during the dry period on mammary gland development. J. Dairy Sci. 94:5976-5986.

Tao, S., I. M. Thompson, A. P. A. Monteiro, M. J. Hayen, L. J. Young, and G. E. Dahl. 2012. Effect of cooling heat-stressed dairy cows during the dry period on insulin response. J. Dairy Sci. 95:50355046

Uyeda, K., and J. J. Repa. 2006. Carbohydrate response element binding protein, ChREBP, a transcription factor coupling hepatic glucose utilization and lipid synthesis. Cell Metab. 4:107-110.

VerHague, M. A., D. Cheng, R. B. Weinberg, and G. S. Shelness. 2013. Apolipoprotein A-IV expression in mouse liver enhances triglyceride secretion and reduces hepatic lipid content by promoting very low density lipoprotein particle expansion. Arterioscler. Thromb. Vasc. Biol. 33:2501-2508.

Vinothkumar, K. R., J. Zhu, and J. Hirst. 2014. Architecture of mammalian respiratory complex I. Nature 515:80-84.

Walker, J. E. 1992. The NADH:ubiquinone oxidoreductase (complex I) of respiratory chains. Q. Rev. Biophys. 25:253-324.

Wheelock, J. B., R. P. Rhoads, M. J. VanBaale, S. R. Sanders, and L. H. Baumgard. 2010. Effects of heat stress on energetic metabolism in lactating Holstein cows. J. Dairy Sci. 93:644-655.

Wiśniewski, J. R., A. Zougman, N. Nagaraj, and M. Mann. 2009. Universal sample preparation method for proteome analysis. Nat. Methods 6:359-362.

Wu, K. C., J. Y. Cui, and C. D. Klaasen. 2011. Beneficial role of Nrf2 in regulating NADPH generation and consumption. Toxicol. Sci. 123:590-600.

Yang, J., S. C. Kalhan, and R. W. Hanson. 2009. what is the metabolic role of phosphoenolpyruvate carboxykinase? J. Biol. Chem. 284:27025-27029.

Zachut, M. 2015. Defining the adipose tissue proteome of dairy cows to reveal biomarkers related to peripartum insulin resistance and metabolic status. J. Proteome Res. 14:2863-2871.

Zachut, M., G. Kra, L. Livshitz, Y. Portnick, S. Yakoby, G. Friedlander, and Y. Levin. 2017. Seasonal heat stress affects adipose tissue proteome toward enrichment of the Nrf2-mediated oxidative stress response in late-pregnant dairy cows. J. Proteomics 158:52-61.

Zachut, M., P. Sood, Y. Levin, and U. Moallem. 2016. Proteomic analysis of preovulatory follicular fluid reveals differentially abundant proteins in less fertile dairy cows. J. Proteomics 139:122-129.

Zhang, Y., and P. A. Edwards. 2008. FXR signaling in metabolic disease. FEBS Lett. 582:10-18. 